\title{
Spherical Model in a Random Field.
}

\author{
A.E. Patrick 1
}

\begin{abstract}
We investigate the properties of the Gibbs states and thermodynamic observables of the spherical model in a random field. We show that on the lowtemperature critical line the magnetization of the model is not a self-averaging observable, but it self-averages conditionally. We also show that an arbitrarily weak homogeneous boundary field dominates over fluctuations of the random field once the model transits into a ferromagnetic phase. As a result, a homogeneous boundary field restores the conventional self-averaging of thermodynamic observables, like the magnetization and the susceptibility. We also investigate the effective field created at the sites of the lattice by the random field, and show that at the critical temperature of the spherical model the effective field undergoes a transition into a phase with long-range correlations $\sim r^{4-d}$.
\end{abstract}

KEY WORDS: Critical fluctuations; disordered spin systems; Gibbs states; selfaveraging.

\section{Introduction.}

The spherical model [5] is a lattice model where a (thermodynamic) random variable $x_{j}$ is attached to every site $j$ of a subset $V_{n}$ of a $d$-dimensional square lattice $Z^{d}$. This model is one of a handful of models where exact results can be obtained in the presence of a random field $\left\{h_{j}, j \in Z^{d}\right\}$. Thermodynamic properties of such a disordered spherical model outside the low-temperature critical line were studied by Pastur in the paper [13]. The magnetization on the critical line was also derived there in the limits $h_{0} \rightarrow \pm 0$, where $h_{0}$ is the expected value of the random field.

Some thermodynamic characteristics have discontinuities on the critical line, and, depending on the boundary conditions and the exact details of passing to the thermodynamic limit $\left(V_{n} \uparrow Z^{d}\right)$, those characteristics can have different limiting values. Their values in the limits $h_{0} \rightarrow \pm 0$ are, in some sense, extreme points of the sets of all possible limiting values. For some models those sets contain simply all linear combinations of the extreme values. For disordered models, like the spherical model in a random field, that is not necessarily the case. The aim of this paper

\footnotetext{
${ }^{1}$ Laboratory of Theoretical Physics, Joint Institute for Nuclear Research, Dubna 141980, Russia e-mail: patrick@theor.jinr.ru
} 
is to study thermodynamic properties of the spherical model directly on the lowtemperature critical line.

Many models in statistical mechanics are complicated enough to force us to restrict the investigation to finding only certain thermodynamic averages. For instance, sometimes investigation of magnetization is reduced to calculation of the averages

$$
\left\langle m_{N}\right\rangle=\frac{1}{N} \sum_{j \in V}\left\langle x_{j}\right\rangle,
$$

where $\langle\cdot\rangle$ denotes the average over the Gibbs distribution. However, as a rule, for a satisfactory understanding of properties of a particular model (especially on a critical line) one has to know distributions of various macroscopic (and, ideally, microscopic) quantities. For that reason in the present paper we study the limiting Gibbs states and the distributions of thermodynamic observables.

One of the properties particular to disordered systems in statistical mechanics is the self-averaging of thermodynamic observables, introduced by Pastur and Figotin in the paper [11]. There they also proved a general theorem concerning the selfaveraging of thermodynamic observables for a wide class of models. By observables they meant quantities already averaged over the Gibbs distribution. For disordered systems involving a (realization of a) random field $\left\{h_{j}, j \in Z^{d}\right\}$ the self-averaging is defined as follows.

Definition 0 (see [11]). A thermodynamic observable $\left\langle Q_{N}\right\rangle$ is self-averaging, if

$$
\lim _{N \rightarrow \infty}\left\langle Q_{N}\right\rangle=Q
$$

exists and is the same for almost all realizations of the random field, where $N$ is the size of the system.

The name self-averaging indicates that one does not have to average the thermodynamic observable $Q_{N}$ over the distribution of the random field. Indeed the limiting distribution is concentrated at the average value, since Eq. (1) trivially implies

$$
\lim _{N \rightarrow \infty}\left\langle Q_{N}\right\rangle=\boldsymbol{E} Q
$$

where $\boldsymbol{E}(\cdot)$ denotes the average over the distribution of the random field. As a rule self-averaging observables are uniformly integrable, see [6, 15], hence, it is also true that

$$
\lim _{N \rightarrow \infty} \boldsymbol{E}\left\langle Q_{N}\right\rangle=Q
$$

From probabilistic point of view there are no fundamental differences between the thermodynamic randomness (described by the Gibbs distribution) and the randomness of the field $\left\{h_{j}, j \in Z^{d}\right\}$. Therefore it seems natural to get rid of the thermodynamic averages in the definition of self-averaging for observables like the magnetization. 
Definition 1. A thermodynamic observable $Q_{N}$ is self-averaging, if

$$
\lim _{N \rightarrow \infty} Q_{N}=Q
$$

exists and is the same for almost all realizations of the random field $\left\{h_{j}, j \in Z^{d}\right\}$, where the limit is understood in probability w.r.t. the thermodynamic randomness.

There are thermodynamic observables which are not self-averaging on critical lines/points, having continuous (non-thermodynamic) distributions. For instance, it is widely known that the susceptibility

$$
\chi_{N}=\frac{1}{N} \sum_{j, k=1}^{N}\left\langle x_{j} x_{k}\right\rangle-\left\langle x_{j}\right\rangle\left\langle x_{k}\right\rangle
$$

is an observable of that kind. On the other hand, there are observables which distributions concentrate at a few (two or more) points. This fact motivated the authors of the paper [3] to introduce the notion of the conditional self-averaging.

Definition 2 (see [3]). A thermodynamic observable $Q_{N}$ is conditionally selfaveraging, if

$$
\lim _{N \rightarrow \infty} Q_{N}-\boldsymbol{E}\left(Q_{N} \mid \xi_{N}\right)=0, \quad \text { in probability, }
$$

where $\boldsymbol{E}\left(\cdot \mid \xi_{N}\right)$ are the conditional averages w.r.t. a sequence of functions of the random field $\left\{\boldsymbol{h}_{j}, j \in Z^{d}\right\}$ which obtain only a finite number of values, $F$, the same for all $N$.

For an illustration of the notion of conditional self-averaging one can look at the random-field Curie-Weiss model, see [3]. In this model a conditionally self-averaging observable $Q_{N}$ is the magnetization $\frac{1}{N} \sum_{j=1}^{N} s_{j}$, the sequence of functions $\xi_{N}$ is the sign of the total random field

$$
\xi_{N}=\operatorname{sgn}\left(\sum_{j=1}^{N} h_{j}\right)
$$

and $\boldsymbol{E}\left(Q_{N} \mid \xi_{N}\right) \sim \xi_{N} m^{*}$, where $m^{*}$ is the spontaneous magnetization.

For a self-averaging observable $Q_{N}$ both thermodynamic (described by the Gibbs distribution) and non-thermodynamic (produced by the random field) fluctuations vanish as $N \rightarrow \infty$. It seems useful to introduce exponents which indicate how fast that happens. The exponent $\rho$, related to non-thermodynamic fluctuations, is defined by

$$
\left\langle Q_{N}-\boldsymbol{E}\left\langle Q_{N}\right\rangle\right\rangle=N^{-\rho} r_{N},
$$

as $N \rightarrow \infty$, where the sequence of random variables $r_{N}$ converges to a random variable with a proper, non-degenerate distribution. The exponent $\tau$, indicating the magnitude of thermodynamic fluctuations, is defined by

$$
Q_{N}-\boldsymbol{E}\left\langle Q_{N}\right\rangle-N^{-\rho} r_{N}=N^{-\tau} t_{N},
$$


as $N \rightarrow \infty$, where, again, the sequence of random variables $t_{N}$ converges to a random variable with a proper, non-degenerate distribution. The definitions of exponents $\rho$ and $\tau$ generalize straightforwardly to the case of conditional self-averaging.

As a rule thermodynamic systems outside critical lines/points are collections of random variables $\left\{x_{j}\right\}_{j=1}^{N}$ with short-range correlations. In this case one usually has self-averaging with the exponents $\rho=\tau=\frac{1}{2}$. More precisely,

$$
m_{N}=\frac{1}{N} \sum_{j=1}^{N} x_{j}=x+N^{-\frac{1}{2}} r_{N}+N^{-\frac{1}{2}} t_{N} .
$$

The exponents $\rho$ and $\tau$ are not fundamentally novel quantities. For most commonly used thermodynamic observables $Q_{N}$ they are related in some way to the standard critical exponents. The values of exponents for the magnetization of the spherical model are calculated in this paper.

Somewhat different terminology was used in the papers [1, 16]. There selfaveraging with exponents $\rho=\tau=\frac{1}{2}$ is called strong self-averaging, while selfaveraging with exponents $\rho \in\left(\frac{1}{2}, 1\right)$ and $\tau \in\left(\frac{1}{2}, 1\right)$ is called weak self-averaging.

Some general results on the behaviour of models under the influence of random field were obtained in the 70s and 80s by application of the renormalization-group ideas to the Ginzburg-Landau model, see [2, 8]. In particular it was noticed that the random-field fluctuations dominate over the thermodynamic fluctuations as the critical point is approached. This observation suggests that the random-field fluctuations also dominate on the low-temperature critical line, and hence one should have $\tau>\rho$ there. This is exactly what happens with the fluctuations of the magnetization of the spherical model, and we will see in Section 6 that in this case $\rho=\frac{1}{2}-\frac{2}{d}$ and $\tau=\frac{1}{2}-\frac{1}{d}$.

The rest of the paper is organized as follows. Section 2 contains the exact definition of the spherical model, the random field and the boundary conditions. It also contains some well known technical results for the use in the later sections. Section 3 summarizes the main results of the paper. In Section 4 we calculate the free energy of the spherical model as an illustration of the application of saddlepoint method in the low-temperature region. In Section 5 we describe in details the properties of the spherical model (the random field $\left\{x_{j}, j \in Z^{d}\right\}$ ) in the infinitevolume limit. In Section 6 we provide an analogous detailed description for the magnetization of the spherical model. The results of Sections 5 and 6 in the absence of the boundary field are re-derived in Section 7. The results of the paper are discussed in Section 8.

\section{The model and useful facts.}

The spherical model describes a collection of random variables $\left\{x_{j}, j \in Z^{d}\right\}$ placed at sites of an integer $d$-dimensional lattice, $Z^{d}$. Every site $j \in Z^{d}$ is specified by its $d$ integer coordinates $\left(j_{1}, j_{2}, \ldots, j_{d}\right)$. 
To define the distribution of random variables at all sites of the lattice, we first specify the joint distribution for the random variables in a finite rectangle

$$
V_{n}=\left\{j \in Z^{d}: 1 \leq j_{\nu} \leq n, \nu=1,2, \ldots, d\right\}
$$

containing $N \equiv n^{d}$ sites, and then pass to the limit $n \rightarrow \infty$. To avoid unnecessary complications we impose periodic boundary conditions in dimensions $2,3, \ldots, d$. Thus the boundary of the rectangle $V_{n}$ is the set

$$
B_{n}=\left\{j \in V_{n}: j_{1}=1, n\right\} .
$$

\section{The Hamiltonian.}

The random variables located in the rectangle $V_{n}$ interact with the boundary field, the external random field, and each other via the Hamiltonian

$$
H_{n}=-J \sum_{j, k \in V_{n}} T_{j k} x_{j} x_{k}-\sum_{j \in V_{n}} h_{j} x_{j}-b \sum_{j \in B_{n}} x_{j},
$$

where $J>0, T_{j k}$ are the elements of the nearest-neighbour interaction matrix, $\left\{h_{j}, j \in Z^{d}\right\}$ is a fixed realization of the external random field, and $b$ is the boundary field.

\section{The interaction matrix.}

The elements of the interaction matrix $\widehat{T}$ are given by

$$
T_{j k}=\sum_{\nu=1}^{d} J^{(\nu)}\left(j_{\nu}, k_{\nu}\right) \prod_{l \in\{1,2, \ldots, d\} \backslash \nu} \delta\left(j_{l}, k_{l}\right),
$$

where

$$
\delta\left(j_{l}, k_{l}\right)= \begin{cases}1, & \text { if } j_{l}=k_{l}, \\ 0, & \text { if } j_{l} \neq k_{l},\end{cases}
$$

is the Kronecker delta.

The coefficients $J^{(1)}\left(j_{1}, k_{1}\right)$ are the elements of the $n \times n$ tri-diagonal matrix

$$
\widehat{J}^{(1)}=\left(\begin{array}{ccccccc}
0 & \frac{1}{2} & & & & & \\
\frac{1}{2} & 0 & \frac{1}{2} & & & 0 & \\
& \frac{1}{2} & 0 & \ddots & & & \\
& & \ddots & \ddots & \ddots & & \\
& & & \ddots & 0 & \frac{1}{2} & \\
& 0 & & & \frac{1}{2} & 0 & \frac{1}{2} \\
& & & & & \frac{1}{2} & 0
\end{array}\right) .
$$


The coefficients $J^{(\nu)}\left(j_{\nu}, k_{\nu}\right)$, for $\nu=2,3, \ldots, d$, are the elements of the matrices $\widehat{J}^{(\nu)}$ which have extra $\frac{1}{2}$ at the upper right and lower left corners (due to the periodic boundary conditions)

$$
\widehat{J}^{(\nu)}=\left(\begin{array}{ccccccc}
0 & \frac{1}{2} & & & & & \frac{1}{2} \\
\frac{1}{2} & 0 & \frac{1}{2} & & & 0 & \\
& \frac{1}{2} & 0 & \ddots & & & \\
& & \ddots & \ddots & \ddots & & \\
& & & \ddots & 0 & \frac{1}{2} & \\
& 0 & & & \frac{1}{2} & 0 & \frac{1}{2} \\
\frac{1}{2} & & & & & \frac{1}{2} & 0
\end{array}\right) .
$$

The eigenvalues of the matrix $\widehat{J}^{(1)}$ are given by

$$
\Lambda_{l}=\cos \frac{\pi l}{n+1}, \quad l=1,2, \ldots, n .
$$

The corresponding orthonormal (that is, orthogonal and normalised) eigenvectors are given by

$$
\boldsymbol{v}^{(l)}=\left\{v_{m}^{(l)}=\sqrt{\frac{2}{n+1}} \sin \frac{\pi l m}{n+1}\right\}_{m=1}^{n}, \quad l=1,2, \ldots, n .
$$

The eigenvalues and orthonormal eigenvectors of the matrices $\widehat{J}^{(\nu)}$, for $\nu=2,3, \ldots, d$, are given by

$$
\lambda_{l}=\cos \frac{2 \pi(l-1)}{n}, \quad l=1,2, \ldots, n,
$$

and

$$
\boldsymbol{u}^{(l)}=\left\{u_{m}^{(l)}=\sqrt{\frac{2}{n}} \cos \left[\frac{2 \pi(l-1)(m-1)}{n}-\frac{\pi}{4}\right]\right\}_{m=1}^{n}, \quad l=1,2, \ldots, n .
$$

Finally, the eigenvalues of the interaction matrix $\widehat{T}$ are the sums of the eigenvalues of the matrices $\widehat{J}^{(\nu)}$

$$
\lambda_{k}=\Lambda_{k_{1}}+\sum_{\nu=2}^{d} \lambda_{k_{\nu}}, \quad k \equiv\left(k_{1}, k_{2}, \ldots, k_{d}\right) \in V_{n} .
$$

The corresponding orthonormal eigenvectors are the products of the eigenvectors of the matrices $\widehat{J}^{(\nu)}$

$$
\boldsymbol{w}^{(k)}=\left\{w_{j}^{(k)}=v_{j_{1}}^{\left(k_{1}\right)} \prod_{\nu=2}^{d} u_{j_{\nu}}^{\left(k_{\nu}\right)}\right\}_{j \in V_{n}}, \quad k \equiv\left(k_{1}, k_{2}, \ldots, k_{d}\right) \in V_{n} .
$$

\section{The external random field.}


We assume that the coefficients $\left\{h_{j}, j \in Z^{d}\right\}$ are a fixed realization of independent normal random variables $\left\{\boldsymbol{h}_{j}, j \in Z^{d}\right\}$ with zero mean and variance $h^{2}$. The assumptions of independence and normal distribution are made to avoid unnecessary complications. The behavior of the model is very different if the random variables $\left\{\boldsymbol{h}_{j}, j \in Z^{d}\right\}$ have, say, Cauchy distribution, or, if the random variables have strong negative correlations severely suppressing fluctuations of sums like $\sum_{j \in V_{n}} \boldsymbol{h}_{j}$. Nevertheless, we restrict our attention to the technically convenient case of independent normal random variables where the fluctuations are neither abnormally large, nor abnormally small.

\section{The Gibbs distribution.}

The distribution of the thermodynamic random variables $\left\{x_{j}, j \in V_{n}\right\}$ is specified by the usual Gibbs density

$$
p\left(\left\{x_{j}, j \in V_{n}\right\}\right)=\frac{e^{-\beta H_{n}}}{\Theta_{n}},
$$

with respect to the spherical "a priori" measure

$$
\mu_{n}(d x)=\delta\left(\sum_{j \in V_{n}} x_{j}^{2}-N\right) \prod_{j \in V_{n}} d x_{j}
$$

The normalization factor (partition function) $\Theta_{n}$ is given by

$$
\Theta_{n}=\int_{-\infty}^{\infty} \cdots \int_{-\infty}^{\infty} e^{-\beta H_{n}} \mu_{n}(d x)
$$

\section{Useful estimates.}

Equations (86)-(12) below state well known results which are used throughout the paper. A routine analysis of the singularity at $\omega_{1}=\omega_{2}=\ldots=\omega_{d}=0$ shows that the function

$$
W_{d}^{(m)}(z) \equiv \int_{-\pi}^{\pi} \ldots \int_{-\pi}^{\pi} \frac{1}{\left(z-\sum_{\nu=1}^{d} \cos \omega_{\nu}\right)^{m}} \prod_{\nu=1}^{d} \frac{d \omega_{\nu}}{2 \pi}<\infty
$$

at $z=d$ if $d>2 m$.

Let $\gamma \in[0,2), \zeta>0$, and $z_{n}=\lambda_{\max }+\zeta n^{-\gamma}$, then we have as $n \rightarrow \infty$

$$
\frac{1}{N} \sum_{k \in V_{n}} \frac{1}{\left(z_{n}-\lambda_{k}\right)^{m}}=W_{d}^{(m)}\left(z_{n}\right)-\frac{1}{2 n} \triangle W_{d}^{(m)}\left(z_{n}\right)+o\left[\exp \left(-n^{1-\gamma / 2} c(\zeta)\right)\right]
$$

where

$$
\triangle W_{d}^{(m)}\left(z_{n}\right) \equiv W_{d-1}^{(m)}\left(z_{n}-1\right)+W_{d-1}^{(m)}\left(z_{n}+1\right)-2 W_{d}^{(m)}\left(z_{n}\right)
$$

and $c(\zeta)$ is strictly positive and increasing for $\zeta>0$. 


$$
\begin{aligned}
& \text { If } \gamma=2, \zeta \geq 0, \text { and } d>4, \text { then } \\
& \frac{1}{N} \sum_{k \in V_{n}}^{\prime} \frac{1}{\lambda_{\max }+\zeta n^{-2}-\lambda_{k}}=W_{d}^{(1)}(d)-\frac{1}{2 n} \triangle W_{d}^{(1)}(d)-\zeta W_{d}^{(2)}(d) n^{-2}+o\left(n^{-2}\right),
\end{aligned}
$$

as $n \rightarrow \infty$, where the prime indicates that the summation does not involve $k=$ $(1,1, \ldots, 1)$.

If $d>2 m$, and $\zeta \geq 0$, then

$$
\frac{1}{N} \sum_{k \in V_{n}}^{\prime} \frac{1}{\left(\lambda_{\max }+\zeta n^{-2}-\lambda_{k}\right)^{m}}=W_{d}^{(m)}(d)+o(1)
$$

as $n \rightarrow \infty$. Approximation of sums of the type (9), (10) by integrals was analysed in [4, 7]. For an outline of a method particularly suited for the above sums see [12].

If $m>0$ and $d>2 m$, then

$$
\int_{-\pi}^{\pi} \ldots \int_{-\pi}^{\pi} \frac{\exp \left(i \sum_{\nu=1}^{d} x_{\nu} \omega_{\nu}\right)}{\left(d-\sum_{\nu=1}^{d} \cos \omega_{\nu}\right)^{m}} \prod_{\nu=1}^{d} \frac{d \omega_{\nu}}{2 \pi} \sim \frac{\Gamma(d / 2-m)}{2^{m} \pi^{d / 2} \Gamma(m)}\left(\sum_{\nu=1}^{d} x_{\nu}^{2}\right)^{m-d / 2}
$$

as $\sum_{\nu=1}^{d} x_{\nu}^{2} \rightarrow \infty$. For a derivation of the above asymptotic formula in the case $m=1$ see, e.g., [9]. The method used in [9] can be also applied in the case $m>0$.

Finally, a direct numerical computation of the multiple integrals $W_{d}^{(m)}(z)$ is an awkward task. Fortunately, for $m>0$ and $z \geq d$, it is reduced to the following integral of the Bessel function $I_{0}(x)$ :

$$
W_{d}^{(m)}(z)=\frac{1}{\Gamma(m)} \int_{0}^{\infty} d v v^{m-1} e^{-z v} I_{0}^{d}(v)
$$

\section{The main results.}

Usually thermodynamic properties are derived in the limit of an infinitely large lattice. In our case the results are most conveniently formulated in a continuum limit. We choose to use the version of continuum limit where the limiting configurations are random functions defined on the $d$-dimensional rectangle $[0,1]^{d}$ :

$$
\{x(\gamma)\}_{\gamma \in[0,1]^{d}} \equiv\left\{x\left(\gamma_{1}, \gamma_{2}, \ldots, \gamma_{d}\right)\right\}_{\gamma_{1}, \gamma_{2}, \ldots, \gamma_{d} \in[0,1]} .
$$

For any $\gamma \in[0,1]^{d}$ the random variable $x(\gamma)$ is defined as the following limit in distribution

$$
x(\gamma) \stackrel{d}{=} \lim _{n \rightarrow \infty} x_{\left(\left[\gamma_{1} n\right],\left[\gamma_{2} n\right], \ldots,\left[\gamma_{d} n\right]\right)},
$$

where $[y]$, is the integer part of $y$.

Thermodynamic random variables $x(\gamma)$ and $x(\delta)$ are limits of the random sequences $x_{\left(\left[\gamma_{1} n\right],\left[\gamma_{2} n\right], \ldots,\left[\gamma_{d} n\right]\right)}$ and $x_{\left(\left[\delta_{1} n\right],\left[\delta_{2} n\right], \ldots,\left[\delta_{d} n\right]\right)}$ separated by a distance of order $n$. Hence, in the continuum limit the random variables $x(\gamma)$ and $x(\delta)$ with $\gamma \neq \delta$ are 
independent due to the exponential/power-law decay of thermodynamic correlations in the high/low temperature region.

Unless explicitly stated otherwise, in this paper we consider dimensions $d \geq 5$ and inverse temperatures $\beta>\beta_{c}$, where $\beta_{c}^{-1}$ is the critical temperature of the spherical model in external random field, see the paper by Pastur [13].

Denote $\varphi_{(1,1, \ldots, 1)}$ the projection of the external random field on the eigenvector $\boldsymbol{w}^{(1,1, \ldots, 1)}$ corresponding to the maximal eigenvalue of the interaction matrix

$$
\varphi_{(1,1, \ldots, 1)}=\sqrt{\frac{2}{n^{d-1}(n+1)}} \sum_{l \in V_{n}} \sin \frac{\pi l_{1}}{n+1} h_{l} .
$$

Recall that the external field $\left\{h_{l}, l \in Z^{d}\right\}$ is a realizations of the random field $\left\{\boldsymbol{h}_{l}, l \in Z^{d}\right\}$ of independent normal random variables with $\boldsymbol{E h}_{l}=0, \boldsymbol{E h}_{l}^{2}=h^{2}$, for any $l \in Z^{d}$. Everywhere below we will use the notation $\mathcal{N}\left(a, b^{2}\right)$ to denote thermodynamic normal random variables with mean $a$ and variance $b^{2}$, which are independent from the external random field. The symbol $q$ will be used to denote a realization of a non-thermodynamic normal random variable $\boldsymbol{q}$. The value of $\boldsymbol{q}$ is fixed once we fix a realization of the random field, and $\boldsymbol{q}$ is always independent of $\mathcal{N}\left(a, b^{2}\right)$.

The main results of the paper can be stated as follows.

1. In the absence of the boundary field, $b=0$, the random variables $x(\gamma)$ have normal distributions with the expected values

$$
\langle x(\gamma)\rangle=\operatorname{sgn}\left[\varphi_{(1,1, \ldots, 1)}\right] \sin \left(\pi \gamma_{1}\right) \sqrt{\left(1-\frac{\beta_{c}}{\beta}\right) \frac{W_{d}^{(1)}(d)}{\beta_{c} J}}+q_{\gamma}
$$

and the variances

$$
\left\langle x^{2}(\gamma)\right\rangle-\langle x(\gamma)\rangle^{2}=\frac{1}{2 \beta J} W_{d}^{(1)}(d),
$$

where $q_{\gamma}$ are independent realizations of zero-mean normal random variables with the common variance

$$
\left(\frac{h}{2 J}\right)^{2} W_{d}^{(2)}(d)
$$

2. For a fixed realization of the external random field, the law of large numbers is valid for the normalized sums

$$
m_{n} \equiv \frac{1}{N} \sum_{j \in V_{n}} x_{j}
$$

as $n \rightarrow \infty$. The convergence to the limiting value can be summarized by the following asymptotic formula

$$
m_{n} \sim \operatorname{sgn}\left[\varphi_{(1,1, \ldots, 1)}\right] \frac{2}{\pi} \sqrt{\left(1-\frac{\beta_{c}}{\beta}\right) \frac{W_{d}^{(1)}(d)}{\beta_{c} J}}+n^{2-d / 2} q_{n}+
$$




$$
+\frac{n^{-d / 4}}{\sqrt{\left|\varphi_{(1,1, \ldots, 1)}\right|}} \mathcal{N}_{n}\left(0, \frac{8}{\pi^{2} \beta} \sqrt{\left(1-\frac{\beta_{c}}{\beta}\right) \frac{W_{d}^{(1)}(d)}{2 \beta_{c} J}}\right),
$$

where $q_{n}$ is a realization of a zero-mean normal random variable with the variance

$$
2 \frac{7 \pi^{2}-69}{3 \pi^{6}}\left(\frac{h}{2 J}\right)^{2} .
$$

Hence, the magnetization $m_{n}$ is (only) conditionally self-averaging with the exponents $\rho=\frac{1}{2}-\frac{2}{d}$ and $\tau=\frac{1}{4}$.

3. For $b \neq 0$ the random variables $x(\gamma)$ have normal distributions with expected values

$$
\langle x(\gamma)\rangle=\frac{b}{J} \frac{\cosh \left[\left(1-2 \gamma_{1}\right) \sqrt{\zeta_{0}}\right]}{\cosh \sqrt{\zeta_{0}}}+q_{\gamma}
$$

and variances

$$
\left\langle x^{2}(\gamma)\right\rangle-\langle x(\gamma)\rangle^{2}=\frac{1}{2 \beta J} W_{d}^{(1)}(d),
$$

where $\zeta_{0}$ is a solution of Eq. (16), and $q_{\gamma}$ are independent realizations of zeromean normal random variables with the common variance

$$
\left(\frac{h}{2 J}\right)^{2} W_{d}^{(2)}(d)
$$

4. For $b \neq 0$, the law of large numbers is valid for the normalized sums

$$
m_{n} \equiv \frac{1}{N} \sum_{j \in V_{n}} x_{j}
$$

as $n \rightarrow \infty$. The convergence to the limiting value can be summarized by the following asymptotic formula

$$
m_{n} \sim \frac{b}{J} \frac{\tanh \sqrt{\zeta_{0}}}{\sqrt{\zeta_{0}}}+n^{2-d / 2} q_{n}+n^{1-d / 2} \mathcal{N}_{n}\left(0, \frac{1}{4 \beta J \zeta_{0}}\left(1-\frac{\tanh \sqrt{\zeta_{0}}}{\sqrt{\zeta_{0}}}\right)\right),
$$

where $q_{n}$ is a realization of a zero-mean normal random variable with the variance (30). Hence, the magnetization $m_{n}$ is self-averaging with the exponents $\rho=\frac{1}{2}-\frac{2}{d}$ and $\tau=\frac{1}{2}-\frac{1}{d}$.

\section{The free energy.}

The calculation of free energy, expected values and correlation functions for the spherical models is reduced, in a routine fashion, to calculation of the large- $n$ asymptotics of an integral. In this section we find the large- $n$ asymptotics for the free energy

$$
f_{n}=-\frac{1}{\beta n^{d}} \ln \Theta_{n} .
$$


A particular attention will be paid to $O\left(n^{-2}\right)$ asymptotics of $f_{n}$, which, as it turns out, determines thermodynamic properties of the model below the critical temperature.

The introduction of new integration variables $\left\{y_{j}\right\}_{j \in V_{n}}$ in Eq. (7) via the orthogonal transformation

$$
x_{j}=\sum_{k \in V_{n}} w_{j}^{(k)} y_{k}, \quad j \in V_{n}
$$

where the eigenvectors $\left\{w_{j}^{(k)}\right\}_{j \in V_{n}}$ are given by Eq. (6) , diagonalises the interaction matrix. Therefore, we obtain the following formula for the partition function

$$
\Theta_{n}=\int_{-\infty}^{\infty} \cdots \int_{-\infty}^{\infty} e^{-\beta \widetilde{H}_{n}(y)} \mu_{n}(d y)
$$

where

$$
\begin{aligned}
\widetilde{H}_{n}(y) & =-J \sum_{k \in V_{n}} \lambda_{k} y_{k}^{2}-\sum_{k \in V_{n}} \varphi_{k} y_{k}-b \sum_{k \in V_{n}} \alpha_{k} y_{k}, \\
\varphi_{k} & =\sum_{j \in V_{n}} h_{j} w_{j}^{(k)}, \quad \text { and } \quad \alpha_{k}=\sum_{j \in B_{n}} w_{j}^{(k)} .
\end{aligned}
$$

Since the vectors $\left\{w_{j}^{(k)}\right\}_{j \in V_{n}}, k \in V_{n}$ are orthonormal, the random variables $\boldsymbol{\varphi}_{k}=$ $\sum_{j \in V_{n}} \boldsymbol{h}_{j} w_{j}^{(k)}$, are independent normal random variables with zero mean and variance $h^{2}$. Therefore, we can treat the coefficients $\varphi_{k}, k \in V_{n}$ as realizations of independent normal random variables.

A direct calculation of the coefficients $\alpha_{k}, k \equiv\left(k_{1}, k_{2}, \ldots, k_{d}\right) \in V_{n}$ (using only the formula for the sum of a geometric series) yields

$$
\alpha_{k}=2 n^{(d-1) / 2} \sqrt{\frac{2}{n+1}} \delta\left(k_{2}, 1\right) \ldots \delta\left(k_{d}, 1\right) \times\left\{\begin{array}{cl}
\sin \frac{\pi k_{1}}{n+1}, & \text { if } k_{1} \text { is odd } \\
0, & \text { if } k_{1} \text { is even }
\end{array}\right.
$$

The integral representation for the delta function

$$
\delta\left(\sum_{j \in V_{n}} y_{j}^{2}-N\right)=\frac{1}{2 \pi i} \int_{-i \infty}^{+i \infty} d s \exp \left[s\left(N-\sum_{j \in V_{n}} y_{j}^{2}\right)\right]
$$

in the "a priori" measure allows one to perform integration over the variables $y_{j}$, $j \in V_{n}$. However, we can switch the order of integration over the variables $y_{j}, j \in V_{n}$ and $s$ only after a shift of the integration contour for $s$ to the right. The shift should assure that the real part of the quadratic form involving the variables $y_{j}, j \in V_{n}$ is negatively defined. The switching of integration order, integration over $y_{j}, j \in V_{n}$, and the introduction of a new integration variable $z$ via $s=\beta J z$ yields

$$
\Theta_{n}=\frac{\beta J}{2 \pi i}\left(\frac{\pi}{\beta J}\right)^{N / 2} \int_{-i \infty+c}^{+i \infty+c} d z \exp \left[N \beta \Phi_{n}(z)\right]
$$


where

$$
\Phi_{n}(z)=J z-\frac{1}{2 \beta N} \sum_{k \in V_{n}} \ln \left(z-\lambda_{k}\right)+\frac{1}{4 J N} \sum_{k \in V_{n}} \frac{\left(\varphi_{k}+b \alpha_{k}\right)^{2}}{z-\lambda_{k}},
$$

and $c>d$ is the shift of the integration contour mentioned above.

The large- $n$ asymptotics of the integral (13) can be found using the saddle-point method. The saddle point of the integrand is a solution of the equation

$$
\Phi_{n}^{\prime}(z)=J-\frac{1}{2 \beta N} \sum_{k \in V_{n}} \frac{1}{z-\lambda_{k}}-\frac{1}{4 J N} \sum_{k \in V_{n}}\left(\frac{\varphi_{k}+b \alpha_{k}}{z-\lambda_{k}}\right)^{2}=0 .
$$

For any $z>d$, as $n \rightarrow \infty$, the sequence of the derivatives $\Phi_{n}^{\prime}(z)$ converges, with probability 1 , to

$$
\Phi^{\prime}(z)=J-\frac{1}{2 \beta} W_{d}^{(1)}(z)-\frac{h^{2}}{4 J} W_{d}^{(2)}(z),
$$

where the functions $W_{d}^{(m)}(z)$ are defined in Eq. (8). The function $\Phi^{\prime}(z)$ increases monotonically with $z$ on $[d, \infty)$, and the location of its zeroes depends on the dimension $d$ of the lattice. Namely, if $d \leq 4$, then the function $\Phi^{\prime}(z)$ has exactly one zero on the interval $[d, \infty)$ at a point $z^{*}>d$, for any $\beta>0$. If $d \geq 5$ and the variance of the external field, $h^{2}$, is sufficiently small, then there exists a critical value

$$
\beta_{c}=\frac{1}{2 J} \frac{W_{d}^{(1)}(d)}{1-\left(\frac{h}{2 J}\right)^{2} W_{d}^{(2)}(d)}
$$

of the parameter $\beta$, see [13]. If $\beta \in\left(0, \beta_{c}\right)$ (the high-temperature regime), then the function $\Phi^{\prime}(z)$ still has exactly one zero on the interval $[d, \infty)$ at a point $z^{*}>d$. While if $\beta>\beta_{c}$ (the low-temperature regime), then the function $\Phi^{\prime}(z)$ is strictly positive on the interval $[d, \infty)$.

The application of the saddle-point method for the integral (13) is fairly straightforward when the saddle point $z^{*}$ is greater than $d$, see [5]. Therefore, in this paper we consider only the low-temperature regime and $d \geq 5$. When $\beta \geq \beta_{c}$, the function $\Phi_{n}(z)$ still attains its minimum on the interval $\left(\lambda_{\max }, \infty\right)$ at a point $z_{n}^{*}>\lambda_{\max }$, where $\lambda_{\max }=d-1+\cos \frac{\pi}{n+1}$ is the maximum eigenvalue of the interaction matrix $\widehat{T}$. However, the sequence of saddle points $z_{n}^{*}$ approaches the branch point of the integrand at $z=\lambda_{\max }$, and the application of the saddle-point method becomes a bit more tricky.

To be able to apply the saddle-point method we have to find a change of variables $z=\lambda_{\max }+\zeta n^{-\gamma}$, such that the sequence of rescaled saddle-points $\zeta_{n}^{*}=\left(z_{n}^{*}-\lambda_{\max }\right) n^{\gamma}$ converges to a positive limit $\zeta^{*}>0$ as $n \rightarrow \infty$. Then, the application of the saddlepoint method for the integral over $\zeta$ becomes straightforward again. Note that the above search for a proper change of variables has an important physical meaning $n^{\gamma / 2} / \sqrt{\zeta^{*}}$ is the correlation length of the model.

In order to find the proper value of $\gamma$ we have to analyse the sums in Eq. (14). The large- $n$ asymptotics of the sum

$$
\Sigma_{1}(z) \equiv \frac{1}{N} \sum_{k \in V_{n}} \frac{1}{z-\lambda_{k}},
$$


when $z=\lambda_{\max }+\zeta n^{-\gamma}$ and $\zeta>0$, follows from Eqs. (9) and (10). Namely, as $n \rightarrow \infty$,

$$
\Sigma_{1}\left(\lambda_{\max }+\zeta n^{-\gamma}\right)=\frac{1}{\zeta n^{d-\gamma}}+W_{d}^{(1)}(d)+O\left(n^{-\min (\gamma, 1)}\right)
$$

To find the large- $n$ asymptotics of the sum

$$
\Sigma_{2}(z) \equiv \frac{1}{N} \sum_{k \in V_{n}} \frac{\varphi_{k}^{2}}{\left(z-\lambda_{k}\right)^{2}}
$$

when $z=\lambda_{\max }+\zeta n^{-\gamma}$, we have to use the law of large numbers. First, we take out the term corresponding to $k=(1,1, \ldots, 1)$ and rearrange the sum as follows

$$
\begin{aligned}
\Sigma_{2}\left(\lambda_{\max }+\zeta n^{-\gamma}\right) & =\frac{\varphi_{(1,1, \ldots, 1)}^{2}}{\zeta^{2} n^{d-2 \gamma}}+\frac{1}{N} \sum_{k \in V_{n}}^{\prime} \frac{h^{2}}{\left(\lambda_{\max }+\zeta n^{-\gamma}-\lambda_{k}\right)^{2}} \\
& +\frac{1}{N} \sum_{k \in V_{n}}^{\prime} \frac{\varphi_{k}^{2}-h^{2}}{\left(\lambda_{\max }+\zeta n^{-\gamma}-\lambda_{k}\right)^{2}}
\end{aligned}
$$

For $\zeta \geq 0$, Eqs. (9) and (11) yield as $n \rightarrow \infty$

$$
\frac{1}{N} \sum_{k \in V_{n}}^{\prime} \frac{1}{\left(\lambda_{\max }+\zeta n^{-\gamma}-\lambda_{k}\right)^{2}}=W_{d}^{(2)}(d)+o(1)
$$

Let $\left\{\xi_{j, n}\right\}_{j=1, n=1}^{\infty}$ be a triangular array of independent random variables with zero expected values. The condition

$$
\sum_{j=1}^{n} \boldsymbol{E}\left|\xi_{j, n}\right|^{s} \rightarrow 0, \quad \text { for some } s \in(1,2],
$$

as $n \rightarrow \infty$, is sufficient for the validity of the law of large numbers

$$
\sum_{j=1}^{n} \xi_{j, n} \rightarrow 0, \quad \text { in probability }
$$

see, e.g., [6]. Therefore Eqs. (8) , (9), and (11) imply

$$
\frac{1}{N} \sum_{k \in V_{n}}^{\prime} \frac{\varphi_{k}^{2}-h^{2}}{\left(\lambda_{\max }+\zeta n^{-\gamma}-\lambda_{k}\right)^{2}} \rightarrow 0, \quad \text { in probability, }
$$

as $n \rightarrow \infty$, if $d>4$, and $\zeta \geq 0$. Summarizing the above we obtain

$$
\Sigma_{2}\left(\lambda_{\max }+\zeta n^{-\gamma}\right)=\frac{\varphi_{(1,1, \ldots, 1)}^{2}}{\zeta^{2} n^{d-2 \gamma}}+h^{2} W_{d}^{(2)}(d)+o(1),
$$

as $n \rightarrow \infty$.

The sum

$$
\Sigma_{3}(z) \equiv \frac{1}{N} \sum_{k \in V_{n}} \frac{\varphi_{k} \alpha_{k}}{\left(z-\lambda_{k}\right)^{2}}
$$


with $z=\lambda_{\max }+\zeta n^{-\gamma}$, is a realisation of a normal random variable with zero mean and the variance

$$
\sigma_{n}^{2}(\zeta)=\frac{1}{N^{2}} \sum_{k \in V_{n}} \frac{h^{2} \alpha_{k}^{2}}{\left(\lambda_{\max }+\zeta n^{-\gamma}-\lambda_{k}\right)^{4}}
$$

It is possible to find a relatively simple expression for the variance

$$
\sigma_{n}^{2}(\zeta)=\frac{2 h^{2}}{n^{d+1}(n+1)} \sum_{k=1}^{n} \frac{\left(1+(-1)^{k+1}\right)^{2} \sin ^{2} \frac{\pi k}{n+1}}{\left(\cos \frac{\pi}{n+1}+\zeta n^{-\gamma}-\cos \frac{\pi k}{n+1}\right)^{4}}
$$

First, note the identity, see [12],

$$
\frac{1}{N} \sum_{k \in V_{n}} \frac{\alpha_{k}^{2}}{z-\lambda_{k}}=\frac{4 x(z)}{n} \frac{x^{n-1}(z)+1}{x^{n+1}(z)+1}
$$

where

$$
x(z)=1+z-d+\sqrt{(z-d)(2+z-d)} .
$$

On differentiating Eq. (15) over $z$ three times we obtain

$$
\begin{gathered}
\frac{1}{N^{2}} \sum_{k \in V_{n}} \frac{\alpha_{k}^{2}}{\left(z-\lambda_{k}\right)^{4}}=\frac{1}{n^{d+1}} \frac{2}{(z-d)(2+z-d)}\left[\frac{1+z-d}{[(z-d)(2+z-d)]^{3 / 2}} \frac{x^{n+1}(z)-1}{x^{n+1}(z)+1}\right. \\
\left.-\frac{4(n+1)^{3} x^{2(n+1)}(z)}{\left(x^{n+1}(z)+1\right)^{4}}+\frac{2(n+1) x^{(n+1)}(z)}{\left(x^{n+1}(z)+1\right)^{2}}\left(\frac{n(n+2)}{3}-\frac{1}{(z-d)(2+z-d)}\right)\right] .
\end{gathered}
$$

Hence, if $\gamma \in(0,2)$, then

$$
\sigma_{n}^{2}(\zeta) \sim \frac{1}{n^{d+1-5 \gamma / 2}} \frac{2 h^{2}}{(2 \zeta)^{5 / 2}}
$$

as $n \rightarrow \infty$, while if $\gamma=2$, then $\lambda_{\max }+\zeta n^{-\gamma} \sim d-\frac{1}{2} \pi^{2} n^{-2}+\zeta n^{-2}$, and $\sigma_{n}^{2}(\zeta) \sim$ $n^{4-d} h^{2} t\left(\zeta-\frac{1}{2} \pi^{2}\right)$, where

$$
t(\zeta)=\frac{1}{\zeta}\left(\frac{1}{(2 \zeta)^{3 / 2}} \tanh \sqrt{\frac{1}{2} \zeta}-\frac{1}{4 \cosh ^{4} \sqrt{\frac{1}{2} \zeta}}+\frac{2 \zeta-3}{12 \zeta \cosh ^{2} \sqrt{\frac{1}{2} \zeta}}\right)
$$

The function $t(\zeta)$ (and similar functions below) has only a removable singularity at $\zeta=0$, and the analytic continuation is to be used for negative values of $\zeta$. Thus

$$
\Sigma_{3}\left(\lambda_{\max }+\zeta n^{-\gamma}\right) \sim O\left(n^{5 \gamma / 4-(d+1) / 2}\right)
$$

does not produce a non-vanishing contribution to the saddle-point equation if $\gamma \leq 2$.

It is also possible to obtain a simple formula for the sum

$$
\Sigma_{4}(z)=\frac{1}{N} \sum_{k \in V_{n}} \frac{\alpha_{k}^{2}}{\left(z-\lambda_{k}\right)^{2}}
$$


by differentiating Eq. (15) over $z$. The differentiation yields

$$
\Sigma_{4}(z)=\frac{8}{n}\left[\frac{x^{n-1}(z)-1}{x^{n+1}(z)+1} \frac{x^{2}(z)}{x^{2}(z)-1}+\frac{(n+1) x^{n+1}(z)}{\left(x^{n+1}(z)+1\right)^{2}}\right] .
$$

On replacing $z$ by $\lambda_{\max }+\zeta n^{-\gamma}$ we obtain

$$
\Sigma_{4}\left(\lambda_{\max }+\zeta n^{-\gamma}\right) \sim\left\{\begin{array}{cl}
\frac{4 n^{-1+\gamma / 2}}{\sqrt{\frac{1}{2} \zeta}}, & \text { if } \gamma \in(0,2) ; \\
\frac{2 \tanh \sqrt{\frac{1}{2}\left(\zeta-\frac{1}{2} \pi^{2}\right)}}{\sqrt{\frac{1}{2}\left(\zeta-\frac{1}{2} \pi^{2}\right)}}+\frac{2}{\cosh ^{2} \sqrt{\frac{1}{2}\left(\zeta-\frac{1}{2} \pi^{2}\right)}}, & \text { if } \gamma=2 .
\end{array}\right.
$$

Thus the sum $\Sigma_{4}(z)$ is dominant among the four sums $\Sigma_{l}(z), l=1,2,3,4$ (if $b \neq 0$ ), in the sense that it is $\Sigma_{4}(z)$ that controls the location of the saddle point $z_{n}^{*}$ in the lowtemperature region. Indeed, the sum $\Sigma_{4}(z)$ produces a non-vanishing contribution to the saddle-point equation already in the scale $z=\lambda_{\max }+\zeta / n^{2}$. Moreover, the extra contribution produced by $\Sigma_{4}(z)$ prevents the rescaled saddle-point $\zeta_{n}^{*}$ approaching the branch-point at $\zeta=0$, where the remaining sums could, potentially, yield nonvanishing contributions to the saddle-point equation.

On introduction of the new integration variable $\zeta$ in Eq. (13) via $z=\lambda_{\max }+\zeta n^{-2}$ we obtain

$$
\Theta_{n}=\frac{\beta J}{2 n^{2} \pi i}\left(\frac{\pi}{\beta J}\right)^{N / 2} \int_{-i \infty+\zeta_{0}}^{+i \infty+\zeta_{0}} d \zeta \exp \left[N \beta \Phi_{n}\left(\lambda_{\max }+\zeta n^{-2}\right)\right] .
$$

The saddle-point of the integrand is $\zeta^{*}=2 \zeta_{0}+\frac{1}{2} \pi^{2}$, where $\zeta_{0}$ is a solution of the equation

$$
1-\frac{1}{2 \beta J} W_{d}^{(1)}(d)-\left(\frac{h}{2 J}\right)^{2} W_{d}^{(2)}(d)=2\left(\frac{b}{2 J}\right)^{2}\left(\frac{\tanh \sqrt{\zeta_{0}}}{\sqrt{\zeta_{0}}}+\frac{1}{\cosh ^{2} \sqrt{\zeta_{0}}}\right) .
$$

Application of the saddle-point method yields

$$
-f_{n}=\frac{1}{\beta n^{d}} \ln \Theta_{n}=\frac{1}{2 \beta} \ln \frac{\pi}{\beta J}+\Phi(d)+n^{-1} \phi_{1}+n^{-2} \phi_{2}\left(2 \zeta_{0}\right)+o\left(n^{-2}\right),
$$

as $n \rightarrow \infty$, where

$$
\begin{aligned}
& \Phi(d)=J d-\frac{1}{2 \beta} L_{d}(d)+\frac{h^{2}}{4 J} W_{d}^{(1)}(d) \\
& L_{d}(z)=\int_{-\pi}^{\pi} \ldots \int_{-\pi}^{\pi} \ln \left(z-\sum_{\nu=1}^{d} \cos \omega_{\nu}\right) \prod_{l=1}^{d} \frac{d \omega_{l}}{2 \pi} \\
& \phi_{1}=\frac{1}{4 \beta} \Delta L_{d}(d)-\frac{h^{2}}{8 J} \Delta W_{d}^{(1)}(d)+\frac{b^{2}}{J} \\
& \phi_{2}(\zeta)=\left(J-\frac{1}{2 \beta} W_{d}^{(1)}(d)-\frac{h^{2}}{4 J} W_{d}^{(2)}(d)\right) \zeta-\frac{b^{2}}{J} \sqrt{2 \zeta} \tanh \sqrt{\frac{1}{2} \zeta}
\end{aligned}
$$


The function $\Phi(z)$ determines the thermodynamics of the model in the hightemperature region. The term $\phi_{1}$ appears because of to the lack of periodicity in one of the dimensions. The function $\phi_{2}(\zeta)$ is responsible for the thermodynamic properties of the model on the low-temperature critical line.

\section{Individual distributions.}

To find the individual distributions of the random variables $\left\{x_{j}, j \in V_{n}\right\}$ we calculate the corresponding characteristic functions

$$
\kappa_{j}(t)=\left\langle\exp \left(i t x_{j}\right)\right\rangle
$$

The saddle-point method described in the previous section yields the following large$n$ asymptotics

$$
\kappa_{j}(t) \sim \exp \left[-\frac{t^{2}}{4 \beta J} \sum_{k \in V_{n}} \frac{\left(w_{j}^{(k)}\right)^{2}}{z_{n}^{*}-\lambda_{k}}+\frac{i t}{2 J} \sum_{k \in V_{n}} \frac{\varphi_{k} w_{j}^{(k)}+b \alpha_{k} w_{j}^{(k)}}{z_{n}^{*}-\lambda_{k}}\right] .
$$

Therefore, for large values of $n$, the individual distributions of the random variables $\left\{x_{j}, j \in V_{n}\right\}$ are nearly normal with mean values

$$
\mu_{j}=\frac{1}{2 J} \sum_{k \in V_{n}} \frac{\varphi_{k} w_{j}^{(k)}+b \alpha_{k} w_{j}^{(k)}}{z_{n}^{*}-\lambda_{k}}
$$

and variances

$$
\sigma_{j}^{2}=\frac{1}{2 \beta J} \sum_{k \in V_{n}} \frac{\left(w_{j}^{(k)}\right)^{2}}{z_{n}^{*}-\lambda_{k}}
$$

On substitution $z_{n}^{*}=d+\zeta^{*} n^{-2}$ one obtains

$$
\sigma_{j}^{2} \rightarrow \frac{1}{2 \beta J} \int_{-\pi}^{\pi} \ldots \int_{-\pi}^{\pi} \frac{1-\cos \left(j_{1} \omega_{1}\right)}{d-\sum_{\nu=1}^{d} \cos \omega_{\nu}} \prod_{l=1}^{d} \frac{d \omega_{l}}{2 \pi}
$$

as $n \rightarrow \infty$. Thus, in the low-temperature region and in the presence of the boundary conditions, $b \neq 0$, the variances of the thermodynamic random variables $x_{j}$ are not affected by the random field $\left\{h_{l}, l \in Z^{d}\right\}$. As $j_{1}$ increases,

$$
\int_{-\pi}^{\pi} \ldots \int_{-\pi}^{\pi} \frac{\cos \left(j_{1} \omega_{1}\right)}{d-\sum_{\nu=1}^{d} \cos \omega_{\nu}} \prod_{l=1}^{d} \frac{d \omega_{l}}{2 \pi} \sim \frac{\Gamma(d / 2-1)}{2 \pi^{d / 2} j_{1}^{d-2}} \rightarrow 0
$$

Hence, only random variables near the boundary have variances noticeably different from the bulk value

$$
\sigma_{\text {bulk }}^{2} \equiv \frac{1}{2 \beta J} W_{d}^{(1)}(d)
$$


The first half of the sum in Eq. (18)

$$
q_{j} \equiv \frac{1}{2 J} \sum_{k \in V_{n}} \frac{\varphi_{k} w_{j}^{(k)}}{z_{n}^{*}-\lambda_{k}}
$$

describes the shift in the expected value of $x_{j}$ due to the external random field. It is a realization of a normal random variable with zero mean and variance

$$
V_{j}^{2} \equiv\left(\frac{h}{2 J}\right)^{2} \sum_{k \in V_{n}}\left(\frac{w_{j}^{(k)}}{z_{n}^{*}-\lambda_{k}}\right)^{2}
$$

As $n \rightarrow \infty$ the variance $V_{j}^{2}$ tends to

$$
\left(\frac{h}{2 J}\right)^{2} \int_{-\pi}^{\pi} \ldots \int_{-\pi}^{\pi} \frac{1-\cos \left(j_{1} \omega_{1}\right)}{\left(d-\sum_{\nu=1}^{d} \cos \omega_{\nu}\right)^{2}} \prod_{l=1}^{d} \frac{d \omega_{l}}{2 \pi}
$$

For $d>4$ we have

$$
\int_{-\pi}^{\pi} \ldots \int_{-\pi}^{\pi} \frac{\cos \left(j_{1} \omega_{1}\right)}{\left(d-\sum_{\nu=1}^{d} \cos \omega_{\nu}\right)^{2}} \prod_{l=1}^{d} \frac{d \omega_{l}}{2 \pi} \sim \frac{\Gamma(d / 2-2)}{4 \pi^{d / 2} j_{1}^{d-4}}
$$

as $j_{1} \rightarrow \infty$. Hence, the variance $V_{j}^{2}$ also approaches its bulk value

$$
V_{\text {bulk }}^{2} \equiv\left(\frac{h}{2 J}\right)^{2} W_{d}^{(2)}(d),
$$

as we move away from the boundary.

The second half of the sum in Eq. (18),

$$
\mu_{j}^{\mathrm{bc}} \equiv \frac{b}{2 J} \sum_{k \in V_{n}} \frac{\alpha_{k} w_{j}^{(k)}}{z_{n}^{*}-\lambda_{k}}
$$

is the shift in the expected value of the thermodynamic random variables $x_{j}$ due to the influence of the boundary conditions. An application of the "contour summation" technique, see [12], yields the following simple formula

$$
\mu_{j}^{\mathrm{bc}}=\frac{b}{J} \frac{x^{n+1-j_{1}}\left(z_{n}^{*}\right)+x^{j_{1}}\left(z_{n}^{*}\right)}{x^{n+1}\left(z_{n}^{*}\right)+1} .
$$

The large- $n$ limit of $\mu_{j}^{\mathrm{bc}}$ depends on the location of the node $j \equiv\left(j_{1}, j_{2}, \ldots, j_{d}\right)$. Assuming $j_{1} \sim \gamma_{1} n$ as $n \rightarrow \infty$, we obtain (recall that $z_{n}^{*}=\lambda_{\max }+\zeta^{*} n^{-2} \sim d+2 \zeta_{0} n^{-2}$ in the low-temperature region, see Eq. (16))

$$
\lim _{n \rightarrow \infty} \mu_{j}^{\mathrm{bc}}=\frac{b}{J} \frac{\cosh \left[\left(1-2 \gamma_{1}\right) \sqrt{\zeta_{0}}\right]}{\cosh \sqrt{\zeta_{0}}} \equiv \mu^{\mathrm{bc}}\left(\gamma_{1}\right) .
$$


The characteristic function of an arbitrary pair $\left(x_{j}, x_{l}\right)$ is given by

$$
\kappa_{j, l}(t, s)=\left\langle\exp \left(i t x_{j}+i s x_{l}\right)\right\rangle \sim \kappa_{j}(t) \kappa_{l}(s) \exp \left[-\frac{t s}{2 \beta J} \sum_{k \in V_{n}} \frac{w_{j}^{(k)} w_{l}^{(k)}}{z_{n}^{*}-\lambda_{k}}\right],
$$

as $n \rightarrow \infty$. Hence, for large values of $n$, the joint distribution of $x_{j}$ and $x_{l}$ is nearly normal with the covariance

$$
\operatorname{cov}\left(x_{j}, x_{l}\right) \sim \frac{1}{2 \beta J} \sum_{k \in V_{n}} \frac{w_{j}^{(k)} w_{l}^{(k)}}{z_{n}^{*}-\lambda_{k}} .
$$

Since $z_{n}^{*}=\lambda_{\max }+\zeta^{*} n^{-2}$, we have (ignoring thin layers near the boundaries)

$$
\lim _{n \rightarrow \infty} \operatorname{cov}\left(x_{j}, x_{l}\right)=\frac{1}{2 \beta J} \int_{-\pi}^{\pi} \ldots \int_{-\pi}^{\pi} \frac{\exp \left[i \sum_{\nu=1}^{d}\left(j_{\nu}-l_{\nu}\right) \omega_{\nu}\right]}{d-\sum_{\nu=1}^{d} \cos \omega_{\nu}} \prod_{\nu=1}^{d} \frac{d \omega_{\nu}}{2 \pi} .
$$

Thus, the covariance $\operatorname{cov}\left(x_{j}, x_{l}\right)$ shows the usual, for the critical line of the ordinary spherical model, power-law decay with the distance $r_{j, l}^{2} \equiv \sum_{\nu=1}^{d}\left(j_{\nu}-l_{\nu}\right)^{2}$ between the nodes $j$ and $l$. Indeed, using Eq. (12) we obtain

$$
\operatorname{cov}\left(x_{j}, x_{l}\right) \sim \frac{\Gamma(d / 2-1)}{4 \beta J \pi^{d / 2} r_{j, l}^{d-2}}
$$

if $1 \ll r_{j, l} \ll n$.

Summarizing, we conclude that the structure of random variables $\left\{x_{j}, j \in V_{n}\right\}$ is fairly simple. Ignoring thin layers near boundaries, we have in the limit $n \rightarrow \infty$

$$
x_{j}=q_{j}+\mathcal{N}_{j}\left(\mu^{\mathrm{bc}}\left(\gamma_{1}\right), \sigma_{\text {bulk }}^{2}\right),
$$

where $q_{j}$ is a realization of a (non-thermodynamic) normal random variable with zero mean and the variance $V_{\text {bulk }}^{2}$ and $\mathcal{N}_{j}\left(a, b^{2}\right)$ is a thermodynamic normal random variable with the mean $a$ and the variance $b^{2}$, see Fig. 1 .

In the presence of the boundary conditions, apart from the global influence through the saddle point $\zeta^{*}$, the external random field $\left\{h_{j}, j \in V_{n}\right\}$ produces only additive contributions (random shifts) $q_{j}$ to the thermodynamic random variables $\left\{x_{j}, j \in V_{n}\right\}$. The properties of the (non-thermodynamic) random variables $\left\{\boldsymbol{q}_{j}, j \in V_{n}\right\}$ generating the shifts are fairly interesting. At the critical temperature $\beta_{c}$ the random field $\left\{\boldsymbol{q}_{j}, j \in V_{n}\right\}$ undergoes a transition into a phase with long-range correlations, see Fig. 2.

Indeed the covariances of the random variables $\left\{\boldsymbol{q}_{j}, j \in V_{n}\right\}$ are given by

$$
\operatorname{cov}\left(\boldsymbol{q}_{j}, \boldsymbol{q}_{l}\right)=\left(\frac{h}{2 J}\right)^{2} \sum_{k \in V_{n}} \frac{w_{j}^{(k)} w_{l}^{(k)}}{\left(z_{n}^{*}-\lambda_{k}\right)^{2}}
$$

Passing to the limit $n \rightarrow \infty$ we obtain

$$
\lim _{n \rightarrow \infty} \operatorname{cov}\left(\boldsymbol{q}_{j}, \boldsymbol{q}_{l}\right)=\left(\frac{h}{2 J}\right)^{2} \int_{-\pi}^{\pi} \ldots \int_{-\pi}^{\pi} \frac{\exp \left[i \sum_{\nu=1}^{d}\left(j_{\nu}-l_{\nu}\right) \omega_{\nu}\right]}{\left(z^{*}-\sum_{\nu=1}^{d} \cos \omega_{\nu}\right)^{2}} \prod_{\nu=1}^{d} \frac{d \omega_{\nu}}{2 \pi}
$$




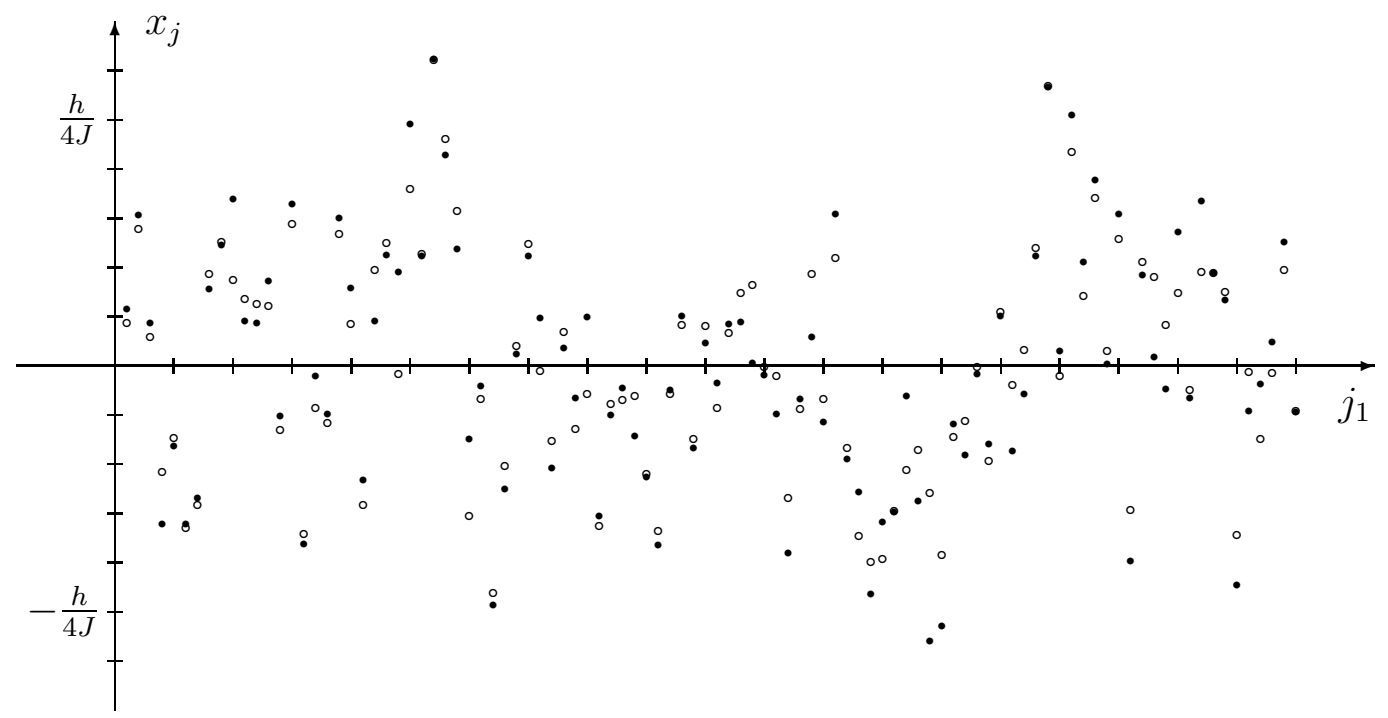

Figure 1: A line of a 5-D realization of thermodynamic random variables $\left\{\boldsymbol{x}_{j}, j \in\right.$ $\left.V_{n}\right\}$ (discs) for $\beta>\beta_{c}$. The picture also contains the corresponding realization of the random field $\left\{\boldsymbol{q}_{j}, j \in V_{n}\right\}$ (circles) driving the random variables at very low temperatures.

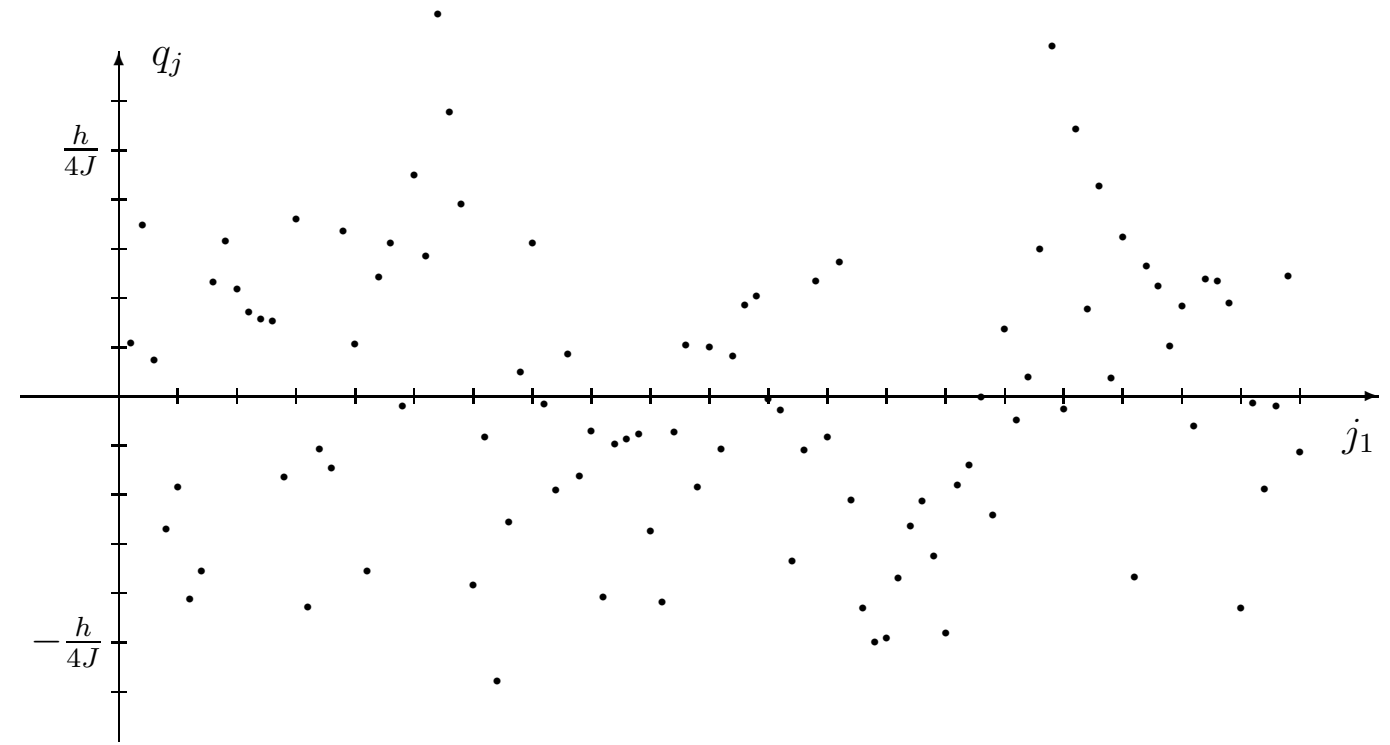

Figure 2: A line of a 5-D realization of the random field $\left\{\boldsymbol{q}_{j}, j \in V_{n}\right\}$ for $\beta>\beta_{c}$. In comparison with independent random variables the field $\left\{\boldsymbol{q}_{j}, j \in V_{n}\right\}$ has a substantial inertia - positive/negative values tend to be surrounded by positive/negative values. 
If $\beta<\beta_{c}$, then $z^{*}>d$ and the above integral decays exponentially with the distance $r_{j, l}$ between the nodes $j$ and $l$. If $\beta \geq \beta_{c}$, then $z^{*}=d$ and Eq. (12) yields the power-law decay

$$
\operatorname{cov}\left(\boldsymbol{q}_{j}, \boldsymbol{q}_{l}\right) \sim \frac{h^{2} \Gamma(d / 2-2)}{16 J^{2} \pi^{d / 2} r_{j, l}^{d-4}} .
$$

Note that the correlations of the random field $\left\{\boldsymbol{q}_{j}, j \in V_{n}\right\}$ decay noticeably slower than the correlations of the thermodynamic random variables, see Eq. (20). This slow decay of the covariances is the reason for the dominance of the random-field fluctuations over the thermodynamic fluctuations.

\section{Macroscopic observables.}

Our aim in this section is to establish the law of large numbers for the normalized sums (magnetization)

$$
m_{n} \equiv \frac{1}{N} \sum_{j \in V_{n}} x_{j},
$$

and to study fluctuations (the central limit theorem) of these sums around the limiting value. The corresponding characteristic functions are given by

$$
\kappa_{n}(t)=\left\langle\exp \left(\frac{i t}{N} \sum_{j \in V_{n}} x_{j}\right)\right\rangle .
$$

The large- $n$ asymptotics of $\kappa_{n}(t)$ is calculated using the technique of the previous section. The saddle-point method yields

$$
\kappa_{n}(t) \sim \exp \left(-\frac{t^{2}}{4 \beta J N^{2}} \sum_{k \in V_{n}} \frac{\eta_{k}^{2}}{z_{n}^{*}-\lambda_{k}}+\frac{i t}{2 J N} \sum_{k \in V_{n}} \frac{\varphi_{k} \eta_{k}+b \alpha_{k} \eta_{k}}{z_{n}^{*}-\lambda_{k}}\right),
$$

where (see Eq. (66))

$$
\eta_{k}=\sum_{j \in V_{n}} w_{j}^{(k)}=n^{(d-1) / 2} \sqrt{\frac{2}{n+1}} \frac{1-(-1)^{k_{1}}}{2} \frac{\sin \frac{\pi k_{1}}{n+1}}{1-\cos \frac{\pi k_{1}}{n+1}} \delta\left(k_{2}, 1\right) \ldots \delta\left(k_{d}, 1\right),
$$

for $k \equiv\left(k_{1}, k_{2}, \ldots, k_{d}\right) \in V_{n}$.

Thus, for large values of $n$, the distribution of the magnetization (21) is approximately normal with the mean value

$$
\mu_{n}=\frac{1}{2 J N} \sum_{k \in V_{n}} \frac{\varphi_{k} \eta_{k}+b \alpha_{k} \eta_{k}}{z_{n}^{*}-\lambda_{k}}
$$

The sum

$$
\frac{1}{2 J N} \sum_{k \in V_{n}} \frac{\varphi_{k} \eta_{k}}{z_{n}^{*}-\lambda_{k}}=\frac{n^{-(d+1) / 2}}{4 J} \sqrt{\frac{2}{n+1}} \sum_{l=1}^{n} \frac{\varphi_{(l, 1,1, \ldots, 1)}\left[1-(-1)^{l}\right]}{1+z_{n}^{*}-d-\cos \frac{\pi l}{n+1}} \frac{\sin \frac{\pi l}{n+1}}{1-\cos \frac{\pi l}{n+1}},
$$


is the shift in the expected value of the magnetization (21) caused by the external random field. It is a realization of a normal random variable with zero mean and the variance

$$
S_{n}^{2} \equiv \frac{h^{2} n^{-d-1}}{4 J^{2}(n+1)} \sum_{l=1}^{n} \frac{1-(-1)^{l}}{\left(1+z_{n}^{*}-d-\cos \frac{\pi l}{n+1}\right)^{2}} \frac{\sin ^{2} \frac{\pi l}{n+1}}{\left(1-\cos \frac{\pi l}{n+1}\right)^{2}} .
$$

On calculating the sum over $l$, see [12], we obtain

$$
S_{n}^{2}=\frac{h^{2} n^{-d-1}}{2 J^{2}\left(z_{n}^{*}-d\right)^{2}}\left[\frac{n}{2}-\frac{2 x^{n+1}\left(z_{n}^{*}\right)+x^{n}\left(z_{n}^{*}\right)-x\left(z_{n}^{*}\right)-2}{\left(x^{n+1}\left(z_{n}^{*}\right)+1\right)\left(x\left(z_{n}^{*}\right)-x^{-1}\left(z_{n}^{*}\right)\right)}+\frac{(n+1) x^{n+1}\left(z_{n}^{*}\right)}{\left(x^{n+1}\left(z_{n}^{*}\right)+1\right)^{2}}\right] .
$$

In the low-temperature region we have $z_{n}^{*}=\lambda_{\max }+\zeta^{*} n^{-2} \sim d+2 \zeta_{0} n^{-2}$, see Eq. (16), therefore

$$
S_{n}^{2} \sim\left(\frac{h}{2 J}\right)^{2} \frac{n^{4-d}}{4 \zeta_{0}^{2}}\left(1-\frac{3 \tanh \sqrt{\zeta_{0}}}{2 \sqrt{\zeta_{0}}}+\frac{1}{2 \cosh ^{2} \sqrt{\zeta_{0}}}\right)
$$

as $n \rightarrow \infty$.

The sum

$$
\frac{b}{2 J N} \sum_{k \in V_{n}} \frac{\alpha_{k} \eta_{k}}{z_{n}^{*}-\lambda_{k}}=\frac{b}{J n} \frac{2 x\left(z_{n}^{*}\right)}{x\left(z_{n}^{*}\right)-1} \frac{x^{n}\left(z_{n}^{*}\right)-1}{x^{n+1}\left(z_{n}^{*}\right)+1},
$$

is the shift in the expected value of the magnetization (21) caused by the boundary conditions. On substitution $z_{n}^{*}=\lambda_{\max }+\zeta^{*} n^{-2}$ one obtains

$$
\frac{b}{2 J N} \sum_{k \in V_{n}} \frac{\alpha_{k} \eta_{k}}{z_{n}^{*}-\lambda_{k}} \sim \frac{b}{J} \frac{\tanh \sqrt{\zeta_{0}}}{\sqrt{\zeta_{0}}}
$$

as $n \rightarrow \infty$.

Let's now look at the variance of the magnetization. According to Eq. (22) it is given by

$$
\sigma^{2} \equiv \frac{1}{2 \beta J N^{2}} \sum_{k \in V_{n}} \frac{\eta_{k}^{2}}{z_{n}^{*}-\lambda_{k}}=\frac{n^{-d-1}}{2 \beta J(n+1)} \sum_{l=1}^{n} \frac{1-(-1)^{l}}{1+z_{n}^{*}-d-\cos \frac{\pi l}{n+1}} \frac{\sin ^{2} \frac{\pi l}{n+1}}{\left(1-\cos \frac{\pi l}{n+1}\right)^{2}} .
$$

The remaining sum over $l$ can be calculated exactly, and we obtain the following expression for the variance

$$
\sigma^{2}=\frac{n^{-d-1}}{2 \beta J\left(z_{n}^{*}-d\right)}\left[n-\frac{2 x\left(z_{n}^{*}\right)\left(x^{n}\left(z_{n}^{*}\right)-1\right)}{\left(x\left(z_{n}^{*}\right)-1\right)\left(x^{n+1}\left(z_{n}^{*}\right)+1\right)}\right] .
$$

On substitution of $z_{n}^{*}=\lambda_{\max }+\zeta^{*} n^{-2}$ for the saddle-point one obtains

$$
\sigma^{2} \sim \frac{n^{2-d}}{4 \beta J \zeta_{0}}\left(1-\frac{\tanh \sqrt{\zeta_{0}}}{\sqrt{\zeta_{0}}}\right)
$$




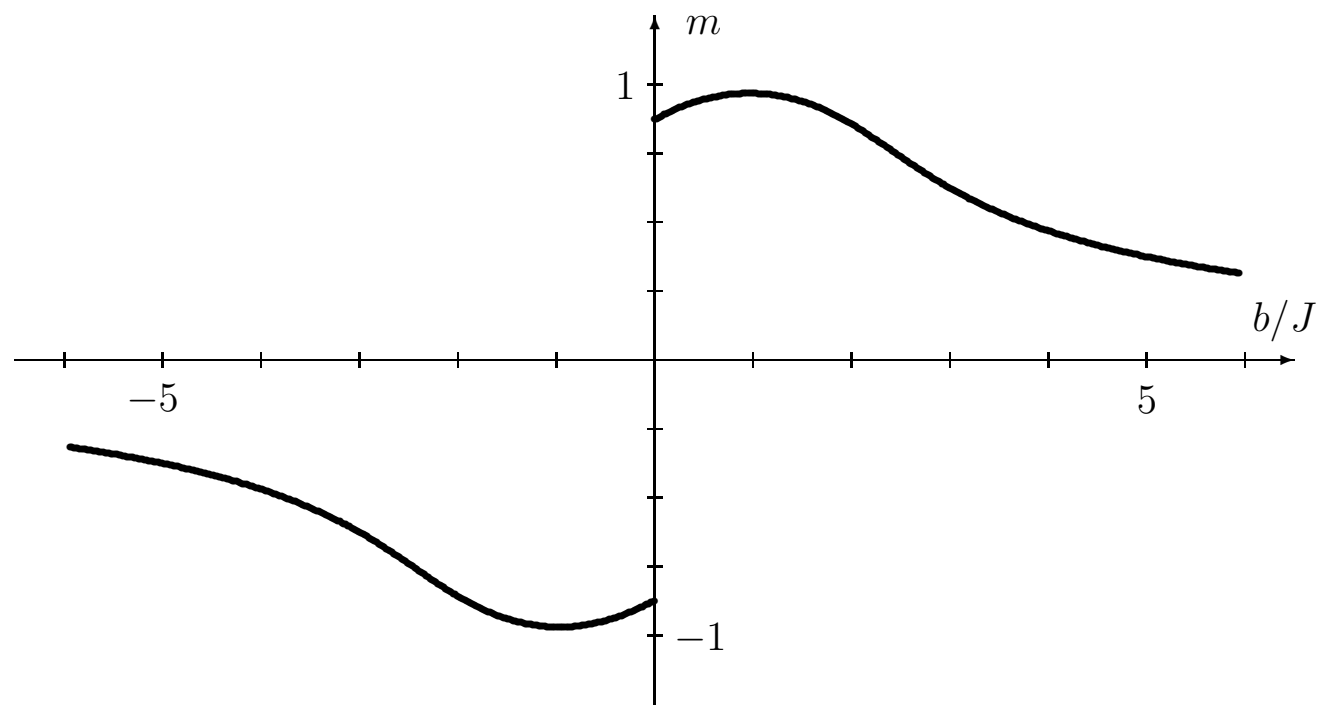

Figure 3: The infinite-lattice magnetization $m=\lim _{n \rightarrow \infty} m_{n}$ as a function of the normalized boundary field $b / J$, for $d=5, \beta J=2$, and $h / J=0.5$. The left $/$ right limits at $b=0$ are given by $\mp \frac{2 \sqrt{2}}{\pi} \sqrt{1-\frac{\beta_{c}}{\beta}}$.

as $n \rightarrow \infty$.

Summarizing the above we obtain the following expression for the magnetization

$$
m_{n} \sim \frac{b}{J} \frac{\tanh \sqrt{\zeta_{0}}}{\sqrt{\zeta_{0}}}+n^{2-d / 2} q_{n}+n^{1-d / 2} \mathcal{N}_{n}\left(0, \frac{1}{4 \beta J \zeta_{0}}\left(1-\frac{\tanh \sqrt{\zeta_{0}}}{\sqrt{\zeta_{0}}}\right)\right)
$$

where $q_{n}$ is a realization of a zero-mean normal random variable with the variance

$$
\left(\frac{h}{2 J}\right)^{2} \frac{1}{4 \zeta_{0}^{2}}\left(1-\frac{3 \tanh \sqrt{\zeta_{0}}}{2 \sqrt{\zeta_{0}}}+\frac{1}{2 \cosh ^{2} \sqrt{\zeta_{0}}}\right)
$$

and $\mathcal{N}_{n}\left(\mu, v^{2}\right)$ is a thermodynamic normal random variable with mean $\mu$ and variance $v^{2}$. Therefore, the magnetization of the spherical model is self-averaging (for $b \neq 0$ ) with the exponents $\rho=\frac{1}{2}-\frac{2}{d}$ and $\tau=\frac{1}{2}-\frac{1}{d}$. The limiting magnetization $m=$ $\lim _{n \rightarrow \infty} m_{n}$ as a function of the boundary field $b$ is shown on Fig. 3.

\section{The distributions for zero boundary field.}

As it is clear from previous sections a non-zero boundary field dominates over the (zero-mean) random field in the low-temperature regime. Therefore in this section we consider the case of zero boundary field.

If $b=0$, then the saddle-point equation for the integral (13) is given by

$$
\Phi_{n}^{\prime}(z) \equiv J-\frac{1}{2 \beta N} \sum_{k \in V_{n}} \frac{1}{z-\lambda_{k}}-\frac{1}{4 J N} \sum_{k \in V_{n}}\left(\frac{\varphi_{k}}{z-\lambda_{k}}\right)^{2}=0 .
$$


Again, the saddle-point $z_{n}^{*}$ drifts towards the branch-point of the integrand in the scale where the terms corresponding to $k=(1,1, \ldots, 1)$ produce a non-vanishing contribution to $\Phi_{n}^{\prime}(z)$. As is obvious from Eq. (31), that happens in the scale $z=\lambda_{(1,1, \ldots, 1)}+\zeta n^{-d / 2}$. The distance from the saddle point $z_{n}^{*}=\lambda_{(1,1, \ldots, 1)}+\zeta^{*} n^{-d / 2}$ to the eigenvalues $\lambda_{k}$ with $k \neq(1,1, \ldots, 1)$ is at least of the order $O\left(n^{-2}\right)$. Therefore there are no additional non-vanishing contribution to the saddle-point equation from those eigenvalues.

In the scale $z=\lambda_{(1,1, \ldots, 1)}+\zeta n^{-d / 2}$ we obtain the following saddle-point equation in the limit $n \rightarrow \infty$

$$
1-\frac{1}{2 \beta J} W_{d}^{(1)}(d)-\left(\frac{h}{2 J}\right)^{2} W_{d}^{(2)}(d)-\frac{1}{4 J^{2}} \frac{\varphi_{(1,1, \ldots, 1)}^{2}}{\zeta^{2}}=0 .
$$

The positive solution of the above equation is given by

$$
\zeta^{*}=\frac{\left|\varphi_{(1,1, \ldots, 1)}\right|}{2 J \sqrt{\left(1-\frac{\beta_{c}}{\beta}\right) \frac{1}{2 J \beta_{c}} W_{d}^{(1)}(d)}} .
$$

The location of the saddle-point $z_{n}^{*}$, as $n \rightarrow \infty$, is given by

$$
z_{n}^{*} \sim d-1+\cos \frac{\pi}{n+1}+\zeta^{*} n^{-d / 2}
$$

Evaluation of the characteristic function (17) at $z_{n}^{*}$ shows that the thermodynamic variables $x_{j}$ have normal distributions with the expected values

$$
\mu_{j}=\frac{1}{2 J} \frac{\varphi_{(1,1, \ldots, 1)} w_{j}^{(1,1, \ldots, 1)}}{z_{n}^{*}-\lambda_{(1,1, \ldots, 1)}}+\frac{1}{2 J} \sum_{k \in V_{n} \backslash(1,1, \ldots, 1)} \frac{\varphi_{k} w_{j}^{(k)}}{z_{n}^{*}-\lambda_{k}}
$$

and variances

$$
\sigma_{j}^{2}=\frac{1}{2 \beta J} \sum_{k \in V_{n}} \frac{\left(w_{j}^{(k)}\right)^{2}}{z_{n}^{*}-\lambda_{k}}
$$

Assuming that $j=\left(j_{1}, j_{2}, \ldots, j_{d}\right)$, and that for $k=1,2, \ldots, d$ we have $j_{k} \sim \gamma_{k} n$ with $\gamma_{k} \in(0,1)$, we obtain

$$
\lim _{n \rightarrow \infty} \mu_{j}=\operatorname{sgn}\left[\varphi_{(1,1, \ldots, 1)}\right] \sin \left(\pi \gamma_{1}\right) \sqrt{\left(1-\frac{\beta_{c}}{\beta}\right) \frac{W_{d}^{(1)}(d)}{\beta_{c} J}}+q_{\gamma}
$$

where $\gamma \equiv\left(\gamma_{1}, \gamma_{2}, \ldots, \gamma_{d}\right)$, and $q_{\gamma}$ are realizations of independent zero-mean normal random variables with the variance $V_{\text {bulk }}^{2}$ given by Eq. (19). An important feature of Eq. (32) is the term $\operatorname{sgn} \varphi_{(1,1, \ldots, 1)}$ common to all expected values $\mu_{j}$. This term is the reason for the absence of conventional self-averaging for the normalized sums (magnetization)

$$
m_{n} \equiv \frac{1}{N} \sum_{j \in V_{n}} x_{j}
$$


On substitution of the saddle point $z_{n}^{*}=\lambda_{(1,1, \ldots, 1)}+\zeta^{*} n^{-d / 2}$ in Eq. (22) we see that, as $n \rightarrow \infty$, the distribution of the magnetization (33) is asymptotically normal with the expected value

$$
\begin{aligned}
\mu_{n} & =\frac{1}{2 J N} \sum_{k \in V_{n}} \frac{\varphi_{k} \eta_{k}}{z_{n}^{*}-\lambda_{k}} \sim \operatorname{sgn}\left[\varphi_{(1,1, \ldots, 1)}\right] \frac{2}{\pi} \sqrt{\left(1-\frac{\beta_{c}}{\beta}\right) \frac{W_{d}^{(1)}(d)}{\beta_{c} J}} \\
& +\frac{1}{2 J N} \sum_{k \in V_{n} \backslash(1,1, \ldots, 1)} \frac{\varphi_{k} \eta_{k}}{z_{n}^{*}-\lambda_{k}} .
\end{aligned}
$$

On subtracting the contribution of the maximum eigenvalue from Eq. (24) one finds that the remaining sum over $k$ is a realization of a normal random variable with zero mean and the variance

$$
S_{n}^{2} \sim 2 \frac{7 \pi^{2}-69}{3 \pi^{6}}\left(\frac{h}{2 J}\right)^{2} n^{4-d},
$$

as $n \rightarrow \infty$.

On substitution of the saddle point $z_{n}^{*}=\lambda_{(1,1, \ldots, 1)}+\zeta^{*} n^{-d / 2}$ in Eq. (27) we find that the thermodynamic variance of the normalized sums (33) is given by

$$
\sigma_{n}^{2} \sim \frac{8}{\pi^{2}} \frac{1}{\left|\varphi_{(1,1, \ldots, 1)}\right| \beta n^{d / 2}} \sqrt{\left(1-\frac{\beta_{c}}{\beta}\right) \frac{W_{d}^{(1)}(d)}{2 \beta_{c} J}} .
$$

as $n \rightarrow \infty$.

Summarizing the above we obtain the following expression for the magnetization

$$
\begin{aligned}
m_{n} & =\operatorname{sgn}\left[\varphi_{(1,1, \ldots, 1)}\right] \frac{2}{\pi} \sqrt{\left(1-\frac{\beta_{c}}{\beta}\right) \frac{W_{d}^{(1)}(d)}{\beta_{c} J}}+n^{2-d / 2} q_{n}+ \\
& +\frac{n^{-d / 4}}{\sqrt{\left|\varphi_{(1,1, \ldots, 1)}\right|}} \mathcal{N}_{n}\left(0, \frac{8}{\pi^{2} \beta} \sqrt{\left(1-\frac{\beta_{c}}{\beta}\right) \frac{W_{d}^{(1)}(d)}{2 \beta_{c} J}}\right)
\end{aligned}
$$

where $q_{n}$ is a realization of a zero-mean normal random variable with the variance

$$
2 \frac{7 \pi^{2}-69}{3 \pi^{6}}\left(\frac{h}{2 J}\right)^{2}
$$

and $\mathcal{N}_{n}\left(0, v^{2}\right)$ is a zero-mean thermodynamic normal random variable with variance $v^{2}$. Thus, in the absence of the boundary field, the magnetization of the spherical model is conditionally self-averaging with the exponents $\rho=\frac{1}{2}-\frac{2}{d}$ and $\tau=\frac{1}{4}$.

\section{Discussion and concluding remarks.}

It was shown in the paper [3] that there are problems with almost sure convergence of Gibbs states for the random-field Curie-Weiss model in the infinite-volume limit. 
In fact, below the critical temperature, the limits of thermodynamic averages $\left\langle s_{j}\right\rangle_{N}$ do not exist, almost surely, as the volume $N$ tends to infinity. A possible solution of the convergence problem was also proposed: it is necessary to consider the limits of distributions of $\left\langle s_{j}\right\rangle_{N}$, which, after some minor technical efforts, lead to correctly defined random infinite-volume Gibbs states. The same problem exists in the spherical model, and, most likely, in such often considered models as the Ising model and $O(n)$ models. Namely, for $b=0, \lim _{n \rightarrow \infty}\left\langle x_{j}\right\rangle_{n}$ does not exist almost surely, although it exists in distribution. The results of the present paper show that switching on a homogeneous boundary field rectifies the problem with almost sure convergence. Namely, for $b \neq 0, \lim _{n \rightarrow \infty}\left\langle x_{j}\right\rangle_{n}$ exist almost surely, which (together with convergence of higher correlation functions) means that the corresponding limit Gibbs state exists for almost all realizations of the random field $\left\{\boldsymbol{h}_{j}, j \in Z^{d}\right\}$.

At approximately the same time, Newman and Stein [10] pointed out that the absence of convergence of local thermodynamic averages, like $\left\langle s_{j}\right\rangle_{N}$, is a natural occurrence in many disordered systems. They call this phenomenon the chaotic size dependence. Somewhat later, Newman and Stein also proposed their own solution of the problem with infinite-volume Gibbs states. Instead of looking at distributions of local averages like $\left\langle s_{j}\right\rangle_{N}$ they choose to look at the empirical distributions

$$
\mathcal{F}_{N}(y) \equiv N^{-1} \#\left\{k \in\{1,2, \ldots, N\}:\left\langle s_{j}\right\rangle_{k} \leq y\right\}
$$

for a fixed realization of randomness. Assuming ergodicity we have

$$
\lim _{N \rightarrow \infty} \mathcal{F}_{N}(y)=\lim _{N \rightarrow \infty} \operatorname{Pr}\left[\left\langle s_{j}\right\rangle_{N} \leq y\right]
$$

hence, both constructions provide the same result: a random infinite-volume Gibbs state. Newman and Stein call the random Gibbs state the metastate.

The authors of the paper [1] investigated self-averaging using the ideas of renormalization group theory. They concluded that there are universality classes of models within which a particular non-self-averaging thermodynamic observable has the same distribution in the thermodynamic limit. The results of the present paper indicate that the conclusion of the paper [1] looks plausible, at least for the magnetization. Indeed, according to Eq. (34) the magnetization of the spherical model obtains the values $\pm m^{*}$ with probability $\frac{1}{2}$, where $m^{*}$ is the spontaneous magnetization. The magnetization of the Curie-Weiss model and, most likely, of disordered finite-dimensional Ising models has the same distribution, see [3]. One can also guess that the magnetization of various disordered $O(n)$ models is uniformly distributed over an $n$-dimensional sphere. On the other hand, we also saw that the distribution of the magnetization is highly-sensitive to symmetry-breaking perturbations. Indeed an arbitrarily weak symmetry-breaking boundary field restores self-averaging, that is, changes a non-degenerate distribution to a degenerate one. Although that fact rather goes along with than contrary to the lines of renormalization group argument.

The susceptibility of the spherical model

$$
\chi_{n}=\beta n^{d}\left[\left\langle\left(\frac{1}{n^{d}} \sum_{j \in V_{n}} x_{j}\right)^{2}\right\rangle-\left(\left\langle\frac{1}{n^{d}} \sum_{j \in V_{n}} x_{j}\right\rangle\right)^{2}\right] \equiv \beta n^{d} \mathrm{t}-\operatorname{Var}\left(m_{n}\right),
$$


can be easily found from Eqs. (29) and (34). If $b \neq 0$, then (when properly normalized) the susceptibility is self-averaging

$$
\chi_{n} \sim \frac{n^{2}}{4 J \zeta_{0}}\left(1-\frac{\tanh \sqrt{\zeta_{0}}}{\sqrt{\zeta_{0}}}\right)
$$

while if $b=0$ then the susceptibility is not a self-averaging observable

$$
\chi_{n} \sim \frac{n^{d / 2}}{\left|\varphi_{(1,1, \ldots, 1)}\right|} \frac{8}{\pi^{2}} \sqrt{\left(1-\frac{\beta_{c}}{\beta}\right) \frac{W_{d}^{(1)}(d)}{2 \beta_{c} J}} .
$$

The susceptibility of various 3D disordered models was studied intensively using Monte-Carlo simulations since mid-90s, see, e.g. [14, 16]. The histograms obtained in [14, 16] suggest that the distribution of the susceptibility is not normal, positively skewed, and has heavy tails. The distribution of the susceptibility given by Eq. (35) has the same properties, and thus, to some extend, explains the results of MonteCarlo simulations. It has been suggested in the paper [1] that the distribution of susceptibility should be the same within universality classes. Since Eq. (35) is the asymptotics of Eq. (27) at the pole $z=\lambda_{\max }$ it is not unreasonable to expect the universality of the distribution of $\chi_{n}$ for a certain class of models. Although it is tempting to speculate that $O(n)$ models might belong to the universality class, nevertheless, the results of the present paper do not indicate neither how wide the universality class is, nor which models possibly belong to this class.

In conclusion, various disordered models have been intensively studied recently either numerically or using various heuristic approaches like, for instance, the renormalization group. The present paper derives explicitly distributions of various thermodynamic quantities within a non-trivial disordered finite-dimensional model the spherical model in a random field. The author hopes that the paper is helpful for understanding the conclusions of heuristic theories, and for interpreting the results of Monte-Carlo simulations.

\section{References}

[1] A. Aharony, and A. B. Harris, Absence of Self-Averaging and Universal Fluctuations in Random Systems near Critical Points, Phys. Rev. Lett. 77:3700-3703 (1996).

[2] A. Aharony, Y. Imry, and S. Ma, Lowering of Dimensionality in Phase Transitions with Random Fields, Phys. Rev. Lett. 37:1364-1367 (1976).

[3] J. Amaro de Matos, A. E. Patrick, and V. A. Zagrebnov, Random infinitevolume Gibbs states for the Curie-Weiss random-field Ising model, J. Stat. Phys. 66:139-164 (1992).

[4] M. N. Barber and M. E. Fisher, Critical phenomena in systems of finite thickness. I. The spherical model, Ann. Phys. 77:1-78 (1973). 
[5] T. H. Berlin and M. Kac, The spherical model of a ferromagnet, Phys. Rev. 86:821-835 (1952).

[6] A. A. Borovkov, Probability theory, (Gordon and Breach Science Publishers, 1998).

[7] M. E. Fisher and V. Privman, First order transition in spherical models: Finitesize scaling, Commun. Math. Phys. 103:527-548 (1986).

[8] Y. Imry, and S. Ma, Random-Field Instability of the Ordered State of Continuous Symmetry, Phys. Rev. Lett. 35:1399-1401 (1975).

[9] E. W. Montroll and G. H. Weiss, Random walks on lattices II, J. Math. Phys. 6:167-181 (1965).

[10] C. M. Newman and D. L. Stein, Multiple states and thermodynamic limits in short-ranged Ising spin-glass models, Phys. Rev. B 46:973-982 (1992).

[11] L. A. Pastur, and A. L. Figotin, On the theory of disordered spin systems, Theor. Math. Phys. 35:403 (1978).

[12] A. E. Patrick, The influence of external boundary conditions on the spherical model of a ferromagnet. I. Magnetization profiles, J. Stat. Phys. 75:253-295 (1994).

[13] L. A. Pastur, Disordered spherical model, J. Stat. Phys. 27:119-151 (1982).

[14] H. Rieger, Critical Behaivior of the Three-Dimensional Random-Field Ising model: Two-Exponent Scaling and Discontinuous Transition, Phys. Rev. B 52:6659-6667 (1995).

[15] A. N. Shiryaev, Probability, (Springer-Verlag, Berlin, 1998).

[16] S. Wiseman, and E. Domany, Lack of Self-Averaging in Critical Disordered Systems, Phys. Rev. E 52:3469-3484 (1995). 